\section{KN-3 Molecular Recognition in Chemical and Biological Systems: A Multi-Dimensional Approach}

François Diederich ${ }^{1}$

\section{ETH Zurich}

email: diederich@org.chem.ethz.ch

We pursue a multi-dimensional approach towards deciphering and quantifying weak intermolecular interactions in chemical and biological systems. Experimental study in this research involves the investigation of protein-ligand interactions, synthetic host-guest complexation, and dynamic processes in designed unimolecular model systems, such as molecular torsional balances. It is complemented by computational analysis and exhaustive data base mining in the Cambridge Crystallographic Database (CSD) and the Protein Data Bank (PDB). Examples of intermolecular interactions quantified by this approach are orthogonal dipolar interactions, organofluorine interactions, stacking on peptide bonds, and halogen bonding. We also investigate the energetics of the replacement of conserved water molecules in protein co-crystal structures by ligand parts. This multi-dimensional approach is illustrated in examples taken from a variety of structure-based drug design projects. Lessons learned are directly applicable to ligand design and optimization in drug discovery and crop protection research, but equally to the assembly of synthetic supramolecular systems. Specific examples will include the replacement of water clusters in protein-ligand complexes of tRNA-guanine transglycosylase (TGT), a target against bacterial shigellosis dysenteriae. It is shown for protein kinase A (PKA), how the glycine-rich loop at the ATP binding site can be favorably addressed by establishing an intense cooperative interaction network. Ligand development against novel targets for antimalarials is illustrated for serine hydroxymethyl transferase (SHMT), a key enzyme from the folate cycle for which ligands had surprisingly not been reported previously. Protein crystallographic work has been essential in all three projects.

Keywords: Molecular Recognition

\section{KN-4 Emphasizing the difference between \\ experimental and theoretical electron}

density in the solid state: new opportunities

from anharmonic thermal motion to excited

states

Birger Dittrich ${ }^{1}$

1. Heinrich-Heine Universität Düsseldorf, Anorganische Chemie und Strukturchemie, Universitätsstraße 1, Gebäude 26.42.01.21, 40225 Düsseldorf

\section{email: bdittri@gwdg.de}

Single-crystal X-ray Bragg diffraction to high scattering angle on suitable, high-quality samples contains detailed information on the electron-density distribution (EDD). Likewise, knowledge of the EDD can be obtained by theoretical methods.

The pseudoatom model [1] has been the method most frequently used for extracting experimental solid-state EDD and coming of age has been proclaimed [2] However, many open questions beyond the well-established EDD topology remain, especially concerning structures so far considered unsuitable for precise experiments on grounds of incomplete de-convolution of displacement parameters and EDD.

Research opportunities related to this situation allow studying (1) temperature dependent changes in structure or EDD (e.g. due to rotational disorder, phase transitions, or dynamic Jahn Teller Distortion/ spin crossover), (2) effects of high-pressure, (3) visible-light excitation, (5) time dependence, (6) anharmonic thermal motion, (7) and/or disorder. Here differences between theory and experiment may be caused by other physical effects. The approximation of using suitable partitioned isolated-molecular ground-state EDD projected onto multipoles ("invarioms") for describing crystal EDD is then often sufficient and permits to calculate differences on a grid, thereby directly showing how experimental signal and theoretical EDD differ for a particular system. Most importantly there is a change in philosophy involved first explored in [4], which could provide an impulse to rejuvenate charge density research: not the EDD but difference densities matter. They should be given more attention.

Examples of applications of this guiding principle will be given. Finally a (literally) exciting new application are "beyond Born-Oppenheimer" experiments, where tunable synchrotron radiation is exploited by probing EDD at and below the absorption edge using otherwise identical measurement conditions, requiring a d-block central atom in a coordination compound. Hard X-ray core-electron excitation/relaxation is faster than nuclear rearrangements, and the difference of the two diffraction patterns contains the information on these ultrafast processes.

[1] Stewart, R. F. (1976). Acta Cryst. A32, 565; Hansen, N. K. \& Coppens, P. (1978). Acta Cryst. A34, 909

[2] Coppens, P. (2005). Angew. Chem. Int. Ed. 44, 6810

[3] Dittrich, B., et al. (2009). Phys. Chem. Chem. Phys. 11,2601

[4] Dittrich, B. et al. (2015) ChemPhysChem, 16, 412 
Keywords: charge density, multipole model, difference electron densities
$\mathrm{KN}-5$ The role of structure and biophysics in the discovery of allosteric kinase inhibitors: ABL001, a potent and specific inhibitor of BCR-ABL

\author{
Sandra W. Cowan-Jacob ${ }^{1}$
}

1. Novartis, Switzerland

email: sandra.jacob@novartis.com

Protein kinases are one of the most successfully targeted protein families with over 30 different FDA-approved drugs on the market and many more undergoing testing in the clinic. Despite this success, there are many challenges to overcome to identify potent, selective inhibitors with good pharmacokinetics. An area that has not yet been exploited widely and that offers opportunities to overcome these challenges is the idea of targeting the kinases outside the ATP site.

Chronic myelogenous leukemia (CML) is characterized by a chromosomal translocation resulting in fusion of the BCR and ABL1 genes and constitutive activation of ABL1 kinase. This fatal disease has been transformed into a chronic condition for a majority of patients due to the discovery of tyrosine kinase inhibitors (TKI), targeting the ATP site of the kinase. However, there remains a subset of patients who develop resistance or who do not tolerate these drugs.

The molecular understanding of the regulation of ABL1 kinase enabled us to design biophysical and biochemical assays that could replicate the effects of blocking the kinase in a physiologically relevant inactive conformation. This resulted in the discovery of ABL001 which binds with single digit $\mathrm{nM}$ affinity to a pocket distant from the ATP site and is active against known clinically relevant mutations in CML. ABL001 is currently being evaluated in Phase 1 clinical trials.

This case study highlights an example of the synergy between biophysics and medicinal chemistry in delivering potentially better drugs, and also demonstrates how an improved molecular understanding of the target can lead to novel modes of targeting proteins.

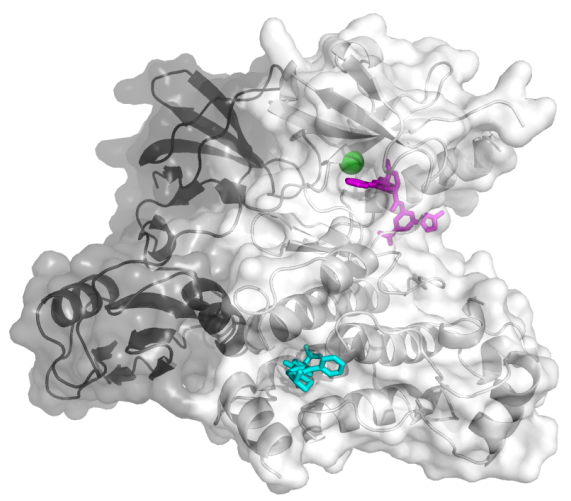

